$X=C[-a, a]$. The operator $K$ is anticommutative with $S$. Indeed,

$$
\begin{gathered}
(S K x)(t)=\int_{-a}^{a} K(-t, s) x(s) d s \\
(K S x)(t)=\int_{-a}^{a} K(t, s) x(-s) d s=-\int_{a}^{-a} K(t,-u) x(u) d u \\
=\int_{-a}^{a} K(t,-u) x(u) d u=-\int_{-a}^{a} K(-t, u) x(u) d u=-(S K x)(t),
\end{gathered}
$$

\title{
A uniform algebra with non-global peak points
}

by

because $K(-t, s)=-K(t,-s)$

Let us consider the equation $\left(a_{0}^{2}-b_{0}^{2} \neq 0 \neq a_{1}^{2}-b_{1}^{2}\right)$

(4.2) $a_{0} x(t)+b_{0} x(-t)+a_{1} \int_{-a}^{a} K(t, s) x(s) d s+b_{1} \int_{-a}^{a} K(-t, s) x(s) d s=y(t)$,

$$
y \epsilon C[-a, a] \text {. }
$$

According to Theorem 2.9, for solving (4.2) it is sufficient to know a solution of the equation $\left(K^{2}-\lambda I\right) x=y$ and all the solutions of the equation $(K-\sqrt{\lambda} I) z_{1}=0$. But

$\left(K^{2} x\right)(t)=\int_{-a}^{a} K_{1}(t, s) x(s) d s, \quad$ where $K_{1}(t, s)=\int_{-a}^{a} K(t, u) K(u, s) d u$.

This means that for solving (4.2) it is enough to solve the following equations :

$$
\begin{aligned}
& -\lambda \tilde{x}(t)+\int_{-a}^{a} K_{1}(t, s) \tilde{x}(s) d s=y(t), \\
& -\sqrt{\lambda} z_{1}(t)+\int_{-a}^{a} K(t, s) z_{1}(s) d s=0 .
\end{aligned}
$$

\section{References}

[1] D. Przeworska-Rolewicz, Sur les équations involutives et leurs applications, Studia Math. 20 (1961), p. 95-117.

[2] - and S. Rolewicz, Equations in linear spaces, Monografie Matematyczno 47, Warszawa 1968 .

[3] - On periodic solutions of linear differential-difference equations with constant coefficients, Studia Math. 32 (1968), p. 69-73.

\section{INSTYTUT MATEMATYCZNY POLSKIEJ AKADEMII NAUK}

INSTITUTE OF MATHEMATICS OF THE POLISH ACADEMYY OF SCTENCES

\author{
Regu par la Rédaction le 8. 7. 1968
}

\section{W. E. MEYERS (Vancouver)}

1. Introduction. By a uniform algebra $A$ on a topological space $X$ we mean an algebra of continuous complex-valued functions on $X$ which contains the constants and is closed under uniform convergence on compact subsets of $X$. A point $p \in X$ is said to be a local peak point of $A$ in $X$ if there exists a neighborhood $U$ of $p$ in $X$ and a function $a \in A$ such that $a(p)=1$ and $|a(x)|<1$ if $x \in U \backslash\{p\}$. If $X$ is compact and the space $M(A)$ of non-zero continuous homomorphisms of $A$, with the $\mathrm{w}^{*}$-topology, is (homeomorphic to) $X$, it is known that every local peak point of $\boldsymbol{A}$ in $X$ is a global peak point, i.e., $U$ can be taken to be $X$ [3]. It is the purpose of this paper to show that this result is not true for general uniform algebras. We exhibit a uniform algebra $A$ on a $\sigma$-compact space $M$ (which is set-wise just the complex numbers $C$ ) which has local peak points in $M=M(A)$ but has no global peak points. In fact, $A$ contains no nonconstant bounded functions.

2. The construction. We describe a sequence of subsets $M_{m}$ of the plane which satisfy

(i) $M_{m} \subset M_{m+1}$;

(ii) $\bigcup_{m=1}^{\infty} M_{m}=C$;

(iii) each $M_{m}$ is compact and non-separating;

(iv) for every $z_{0}$ on the positive $y$-axis, there is a sequence $\left\{z_{m}\right\}_{m=1}^{\infty}$, $z_{m} \in M_{m}-M_{m-1}$, such that $z_{m} \rightarrow z_{0}$;

(v) for every positive integer $m$ and every $z_{0}$ on the positive $y$-axis with $\left|z_{0}\right| \leqslant m$ there is a sequence $\left\{z_{n}\right\}_{n=1}^{\infty} \subset M_{m}$ such that $z_{n} \rightarrow z_{0}$;

(vi) for every $z$ not lying on the positive $y$-axis, $z$ lies in the interior int $M_{m}$ of $M_{m}$ for some $m$.

Let $B_{m}$ be the union of the lines $\{(1 / n, y): m / 2 n \leqslant y \leqslant m\}$ and the line segments joining the following pairs of points:

$$
(0,-m) \text { and }(m,-m) ;(m,-m) \text { and }(m, m) \text {; }
$$




$$
\begin{gathered}
(m, m) \text { and }\left(\frac{1}{m}, m\right) ; \quad\left(\frac{1}{m}, m\right) \text { and }\left(\frac{1}{m}, \frac{1}{2}\right) ; \\
\left(\frac{1}{m}, \frac{1}{2}\right) \text { and }(0,0) ; \quad(0,0) \text { and }(0, m) .
\end{gathered}
$$

Take $M_{m}$ to be the closed and bounded subset of $C$ bounded by $B_{m}$ and its reflection about the $y$-axis.

For each $m$, let $A_{n}=\left(P \mid M_{m}^{-}\right)$be the uniform closure on $M_{m}$ of the algebra $P$ of polynomials in $z$. By a well-known theorem of Mergelyan, $\boldsymbol{A}_{m}$ is the commutative Banach algebra of all continuous functions on $M_{m}$ which are analytic on int $M_{m}$. Let $\pi_{j}^{m}: A_{m} \rightarrow A_{j}, m \geqslant j$, be the algebra isomorphism given by $\pi_{j}^{m}\left(f_{m}\right)=f_{m} \mid M_{j}, f_{m} \in A_{m}$. The collection $\left\{A_{m}, \pi_{j}^{m}\right\}$ determines a dense inverse limit system [1]; Let $A$ be the inverse limit of this system and write $\pi_{m}$ for the canonical projection of $A$ into $\boldsymbol{A}_{m}$. Then $\boldsymbol{A}$ is a commutative $\boldsymbol{F}$-algebra whose non-zero continuous homomorphism space $M$ is $\sigma$-compact and can be identified set-wise with $C$. Further, $A$ can be regarded as a uniform algebra on $M$ with $M(A)=M$ by defining $f(z)=\pi_{m}(f)(z), f \epsilon A, z \in M_{m}$.

Birtel and Lindberg show in [2] that an algebra $A$ defined in this way contains no bounded functions except constants, so of course no point of $M$ can be a (global) peak point of $A$ in $M$. However, if $q=i y$, $y>1$, then $q$ is a local peak point of $A$ in $M$, as the following lemmas show.

LeMra 1. Let $q=i y_{0}, y_{0}>1$, and take an integer $r$ with $y_{0}<r-1$ and $\left|y_{0}-1\right|>1 / r$. Let

$$
K_{m}=\left\{(x, y): \frac{4 m+7}{4(m+1)(m+2)} \leqslant|x| \leqslant \frac{4 m+1}{4 m(m+1)}, 1 \leqslant y \leqslant r\right\}
$$

and let $K$ be the closure in $C$ of $\bigcup_{m=1}^{\infty} K_{m}$. Then there is a neighborhood $U$ of $q$ in $M$ with $U \subset K$.

Proof. Set

$$
Y_{m}=\left\{(x, y): \frac{4 m+1}{4 m(m+1)} \leqslant|x| \leqslant \frac{4 m+3}{4 m(m+1)}, 1 \leqslant y \leqslant m\right\} .
$$

Then $Y_{m}$ is compact, $Y_{m} \cap M_{m}=\varnothing$, and $Y_{m} \subset M_{m+1}$. We find for each $m$ a polynomial $p_{m}$ as follows: Let

$$
f_{1}(z)= \begin{cases}2, & z \epsilon Y_{1} \\ 0, & z \in M_{1}\end{cases}
$$

By Mergelyan, there is a polynomial $p_{1}$ with $\left\|p_{1}-f_{1}\right\|_{M M_{1} \cup Y_{1}}<\frac{1}{4}$. Assume now that $p_{m-1}$ has been chosen. Since

$$
f_{m}(z)=\left\{\begin{array}{cc}
p_{m-1}(z), & z \in M_{m}, \\
2, & z \in Y_{m},
\end{array}\right.
$$

is continuous on $M_{m} \cup Y_{m}$ and analytic on the interior of this set, there is a polynomial $p_{m}$ such that $\left\|p_{m}-f_{m}\right\|_{M_{m} \cup F_{m}}<1 / 2^{m+1}$. If $\varepsilon>0$ and $m$ is taken large enough so that $\sum_{k=m}^{\infty} \frac{1}{2^{k+2}}<\varepsilon$, then for $n>m$,

$$
\begin{aligned}
\left\|p_{n}-p_{m}\right\|_{M_{m}} & \leqslant \sum_{k=m}^{n-1}\left\|p_{k+1}-p_{k}\right\|_{M M_{m}} \leqslant \sum_{k=m}^{n-1}\left(\left\|p_{k+1}-f_{k+1}\right\|_{M_{m}}+\left\|f_{k+1}-p_{k}\right\|_{M_{m}}\right) \\
& <\sum_{k=m}^{n-1} \frac{1}{2^{k+2}}<\varepsilon
\end{aligned}
$$

so $\left\{p_{n}\right\}_{n=1}^{\infty}$ converges uniformly on each set $M_{m}$ and thus determines an element $f \in A$. But

$$
|f(q)| \leqslant\left|p_{1}(q)\right|+\sum_{k=1}^{\infty}\left|p_{k+1}(q)-p_{k}(q)\right|<\frac{1}{4}+\sum_{k=1}^{\infty} \frac{1}{2^{k+2}}<1
$$

while if $z \epsilon Y_{m}$ for some $m$, then

$$
|f(z)| \geqslant\left|p_{m}(z)\right|-\sum_{k=1}^{\infty}\left|p_{k+1}(z)-p_{k}(z)\right|>\left(2-\frac{1}{2^{m+1}}\right)-\sum_{k=m}^{\infty} \frac{1}{2^{k+2}}>1 .
$$

Thus if $Y=\bigcup_{m=1}^{\infty} Y_{m}$, then $\{z \in M:|f(z)|<1\} \frown Y=\varnothing$, so the $\mathrm{w}^{*}$-open neighborhood $U=\{z:|f(z)|<1,|z-q|<1 / r\}$ of $q$ in $M$ is contained in $K$ since $K \cup Y \supset\{z:|z-q|<1 / r\}$.

LEMMA 2. There is an element $a \in A$ which peaks at $q$ in $K$.

Proof. Set $X_{m}=M_{m} \cup K$ and let $U_{m}=\{z:|z-q|<1 / m\}$. Note that $X_{m}$ is a compact non-separating subset of $C$. We find by induction polynomials $b_{m}$ which satisfy

$$
\begin{gathered}
\left\|b_{m+1}-b_{m}\right\|_{X_{m}}<\frac{1}{2^{m-1}} \\
\left\|b_{m+1}-b_{m}\right\|_{K \mid U_{m}}<\frac{1}{2^{m+1}} \\
b_{m}(q)=3\left(1-\frac{1}{2^{m}}\right) \\
\left\|b_{m}\right\|_{K} \leqslant 3\left(1-\frac{1}{2^{m+1}}\right)
\end{gathered}
$$

Studia Mathematica XXXIII.2 
By Mergelyan, there is a function $h_{m} \epsilon\left(P \mid X_{m}\right)$ which peaks in $X_{m}$ at $q$. Let $\varphi_{m}$ be a conformal map of int $M_{m}$ onto $\left\{z:|z|<\frac{1}{2}\right\}$ and $\varphi_{0}$ a conformal map of the right half-plane $H$ onto $\{z:|z|<1\}$ with continuous boundary values taken so that $\varphi_{0}(q)=1$.

Write

$$
h_{m}(z)= \begin{cases}\varphi_{m}(z), & z \in \operatorname{int} M_{m}, \\ \varphi_{0}(z), & z \epsilon K_{n} \cap H, n>m, \\ \varphi_{0}\left(z+\frac{1}{n}\right), & z \epsilon K_{n} \backslash H, n>m,\end{cases}
$$

and extend $h_{m}$ to a function continuous on $X_{m}$ with $\left|h_{m}(z)\right|<1$ for $z \in M_{m} \backslash\{q\}$.)

Let $a_{1} \epsilon P$ be such that $\left\|a_{1}-f_{1}\right\|_{X_{1}}<\frac{1}{8}$ and set $b_{1}=\frac{3}{2}\left(a_{1} / a_{1}(q)\right)$. Then $\left\|b_{1}\right\|_{K}<\frac{3}{2}\left(1+\frac{1}{8}\right) /\left(1-\frac{1}{8}\right) \leqslant 3\left(1-\frac{1}{4}\right)$ and $b_{1}(q)=3\left(1-\frac{1}{2}\right)$. Suppose $b_{1}, \ldots, b_{k}$ have been picked satisfying $(1)-(4)$. Since $b_{k}(q)=3\left(1-1 / 2^{k}\right)$, there is some $j>k$ such that

$$
\left|b_{k}(z)\right|<3\left(1-\frac{1}{2^{k}}\right)+\frac{1}{2^{k+2}} \quad \text { for } z \epsilon U_{j} .
$$

Choosing $n_{j}$ large enough so that $\left\|h_{j}^{n j}\right\|_{X_{j} \mid U_{j}}<\frac{1}{16}$ and $a_{j} \in P$ with $\left\|a_{j}-h_{j}^{n j}\right\|_{X_{j}}<\frac{1}{16}$, set $g_{j}=a_{j}-\frac{1}{8}$.

Then $\left\|g_{j}\right\|_{X_{j}}<1,\left\|g_{j}\right\|_{X_{j} \mid U_{j}}<\frac{1}{4}$, and $\left|g_{j}(q)\right|>\frac{3}{4}$. Let

$$
b_{k+1}=b_{k}+\frac{3}{2^{k+1}} \frac{g_{j}}{g_{j}(q)} .
$$

It is easily checked that $b_{k_{+1}}$ satisfies (1), (2), and (3). To see (4), note that if $z \in K \cap U_{j}$,

$$
\left|b_{k+1}(z)\right| \leqslant 3\left(1-\frac{1}{2^{k}}\right)+\frac{1}{2^{k+2}}+\frac{3}{2^{k+1}} \cdot \frac{4}{3}=3\left(1-\frac{1}{2^{k+2}}\right)
$$

while if $z \epsilon K \backslash U_{j}$,

$$
\left|b_{k+1}(z)\right| \leqslant 3\left(1-\frac{1}{2^{k+1}}\right)+\frac{3}{2^{k+1}} \cdot \frac{4}{3} \cdot \frac{1}{4}<3\left(1-\frac{1}{2^{k+2}}\right) .
$$

Thus we have by induction the desired polynomials $b_{m}$. By (1), $\left\{b_{m}\right\}_{m=1}^{\infty}$ converges uniformly on the sets $M_{m}$ to $b \in A$. By $(3), b(q)=3$. If $z \epsilon K \backslash\{q\}$, then there exists $m$ such that $z \epsilon{D_{m}}_{m}$, so by (2) and (4)

$|b(z)| \leqslant\left|b_{m}(z)\right|+\sum_{k=m}^{\infty}\left|b_{k+1}(z)-b_{k}(z)\right| \leqslant 3\left(1-\frac{1}{2^{m+1}}\right)+\frac{1}{2^{m}}=3-\frac{1}{2^{m+1}}<3$.
Thus $a=b / 3$ peaks at $q$ in $K$ and the lemma is proved.

That $q$ is a local peak point of $A$ in $M$ (where $q=i y, y>1$ ) now follows immediately since the $w^{*}$-neighborhood $U$ of $q$ is contained in $K$.

\section{References}

[1] R. Arens, Dense inverse limit rings, Mich. Math. J. 5 (1958), p. 169-182. [2] F. Birtel and J. A. Lindberg, A Liouville algebra of non-entire funetions, Studia Mathematica 25 (1964), p. 27-31.

[3] H. Rossi, The local maximum modulus principle, Ann. Math. (2) 72 (1960), p. $1-11$.

Reģu par la Rédaction le 15. 8. 1968 